\title{
Heterozygous genotype
}

National Cancer Institute ( $\mathrm{NCl})$

\section{Source}

National Cancer Institute (NCI). Heterozygous genotype.

Occurs when the two alleles at a particular gene locus are different. A heterozygous genotype may include one normal allele and one mutation, or two different mutations. The latter is called a compound heterozygote. 